\title{
Publics souhaités, publics refusés : les publics comme critères de la valeur de l'œuvre dans les années 1550
}

FLORENCE BONIFAY

Université Lumière-Lyon 2

Dans le contexte des tensions poétiques exacerbées des années 1550, les lectorats circonscrits par les auteurs sont manipulés comme arguments pour construire la valeur d'une ouvre ou lui ôter tout crédit. Les poètes novateurs et leurs adversaires s'emploient ainsi à désigner les publics qu'ils prétendent avoir, à rejeter les publics qu'ils estiment indignes et à se refuser mutuellement des publics de valeur. Ces débats, par écrits interposés, nous donnent des indices pour mieux comprendre les échelles de valeur alors en discussion, non seulement à propos des œuvres mais aussi des publics. Les pairs, les doctes, les têtes couronnées ou encore les femmes apparaissent ainsi comme des lectorats plus ou moins légitimants selon les différents points de vue des auteurs.

In the context of the exacerbated poetic tensions of the 1550s, the readerships defined by authors are manipulated to serve as arguments in constructing the merits of a work or in discrediting it. Innovative poets and their opponents thereby work to designate the audiences they claim to have, to reject those they deem unworthy, and to deny one another valuable readership. These debates, embedded in the texts, offer clues towards a better understanding of the measures of value being discussed at the time, not only in terms of the texts but also in terms of audiences. Peers, erudite men, royalty, or even women appear as more or less legitimizing readerships, depending on the authors' different points of view.

O comble de grands liesses!

Si le Princé à qui t'adresses

Lit tes œuvres d'un bon œil :

Si la digne recompense

Respond à ta diligence,

Acomply sera ton vueil.

Ceste œuvre au moins proufitable

Te sera, si delectacle

Elle est aux joyeux lecteur [sic] :

Car l'esprit humain se baigne

Se sentant priser, \& gaigne

Ce bien duquel est autheur.

Mais si le lecteur mesprise 
Ceste histoire tant exquise,

Je dixay que c'est malheur :

Puis que labeur \& paresse,

Puis qu'avarice \& largesse

Ont mesme pris \& valeur. ${ }^{1}$

Ces trois derniers sizains d'une ode de Jean-Pierre de Mesmes, rédigée pour soutenir la publication, en 1550, d'une traduction de L'Histoire de Primaléon de Grece par François de Vernassal, sont le lieu d'une réflexion sur le lectorat et l'appréciation de la valeur de l'œuvre. Trois types de publics y sont hiérarchisés : le roi, les « joyeux lecteur[s] » et les lecteurs méprisants. Le regard approbateur du premier comblerait ( «au comble») les attentes les plus ardentes de l'auteur en donnant une pleine légitimité à son œuvre ; l'appréciation des seconds occasionnerait " au moins » une satisfaction dont l'auteur pourrait tirer profit, ne serait-ce qu'en termes de reconnaissance ; le dédain des derniers serait simplement le signe de leur manque de jugement sur ce qui fonde la " valeur » d'une œuvre. Quand il s'agit de faire rimer " autheur » (v. 30) et «valeur» (v. 36), ces trois types de « lecteur[s]» (v. 27) sont donc plus ou moins qualifiés et qualifiants. C'est à cet usage du public dans les débats des années 1550 sur la valeur des œuvres que nous voulons consacrer le présent propos.

De fait, il nous semble que les périodes de discussion, voire de conflit, sur ce qui fonde la valeur des œuvres offrent un terrain fertile à la réflexion sur le lectorat. Les sizains de Jean-Pierre de Mesmes articulent par exemple la réflexion sur les lecteurs de la traduction de Vernassal à une hypothèse de réception négative. Peut-être l'encomiaste pense-t-il, entre autres, à la condamnation récente par Du Bellay du roman et des traductions dans La Deffence, et illustration de la langue françoyse. Toutefois, l'allusion ne peut être que très vague ou la tension que passagère, attendu que de Mesmes et Vernassal sont nommés amicalement dans les vers de Ronsard, Baiff ou Magny, au cours des années 1550 . Au-delà de ce cas particulier, nous consacrerons une première

1. Jean-Pierre de Mesmes, "Ode de J.P.D.M. à Vernassal ", in L'Histoire de Primaleon de Grece continuant celle de Palmerin d'Olive Empereur de Constantinople son père, naguere tirée tant de l'Italien comme de l'Espagnol, \& misł en nostre vulgaire par François de Vernassal Quercinois (Paris : Estienne Groulleau, 1550, exemplaire de la Bibliothèque nationale d'Autriche, 80.P.13), v. 19-36, fol. a 6 vº. Dans cet article, quand notre source est une édition de la Renaissance, nous en respectons la typographie, avec tous les signes diacritiques. 
partie à étayer l'hypothèse que le public et la notion de bien public surgissent comme argument, principalement lorsque la valeur d'une œuvre fait débat, en observant la période de production poétique qui s'étend des années 1550 (avènement de la génération Ronsard) à 1570 (guerres de religion et arrivée d'une nouvelle génération poétique). Notre deuxième partie se focalisera plus précisément sur les années 1550, tant l'arrivée d’une nouvelle génération de poètes - désireux de s'imposer d'emblée au sommet du Parnasse - nourrit d'âpres discussions, par écrits interposés, sur ce que valent les lettres et plus spécifiquement la poésie, notamment amoureuse. Dans ces débats, la valeur est en partie indexée à la qualité du public visé, qui devient une sorte de miroir de la qualité de l'œuvre. Les années 1550 , traversées de fortes tensions à propos de la valeur des œuvres, nous semblent ainsi propices à observer des constructions de publics souhaités ou rejetés.

\section{De l'« utilité publique » au public utile : \\ le public comme critère de valeur de l'œuvre}

\section{1) Publication et utilité publique}

Issues en partie des écrits d'Aristote (Éthique à Nicomaque; Politique) et de Cicéron (De officiis; De senectute), christianisées par Albert le Grand (Super Ethica) et saint Thomas d'Aquin (commentaire sur l'Éthique; De regimine principum) mais aussi adaptées à un régime monarchique par les hommes du Moyen Âge 2 , les notions d'utilitas communis, d'utilitas publica, de communis profectus et de bonum publicum croisent, à la Renaissance, la réflexion des autorités royales sur le rôle des auteurs. Aux yeux d'un Prince à qui est confié la gestion de l'intérêt commun ainsi que la recherche de l'utilité de chacun et de tous $^{3}$, certains auteurs peuvent participer à la cohésion nationale en affectant

2. Voir le numéro thématique Pouvoir d'un seul et bien commun (VI $-X V I^{e}$ siècles) de la Revue française d'histoire des idées politiques 32.2 (2010), et notamment l'article de Bénédicte Sère « Aristote et le bien commun au Moyen Âge : une histoire, une historiographie » : 277-291. Voir aussi Hervé Oudart, JeanMichel Picard et Joëlle Quaghebeur, éd., Le Prince, son peuple et le bien commun de l'Antiquité tardive à la fin du Moyen Âge (Rennes : Presses universitaires de Rennes, 2013).

3. Arlette Jouanna rappelle que la notion d'utilité publique, tant concrète qu'abstraite sous la plume des penseurs de la Renaissance, esquisse une ébauche du concept d'État : Arlette Jouanna, Le Pouvoir absolu. Naissance de l'imaginaire politique de la royauté (Paris : Gallimard, 2013), 40. 
leurs œuvres ad communem utilitatem par le biais de la publication imprimée. En introduction d'une récente anthologie critique de Privilèges d'auteurs et d'autrices en France (XVI $I^{\mathrm{e}} \mathrm{XVII^{e }}$ siècles), Michèle Clément et Edwige KellerRahbé soulignent ainsi que le privilège est le lieu stratégique d'une valorisation de l'utilité publique de l'auteur. Cela s'observe notamment dans les privilèges de la seconde moitié du XVI ${ }^{e}$ siècle :

De l'autorité pensée exclusivement comme droit (ce qui prévaut surtout entre 1505 et 1545), combinant une clause juridique et une clause économique (exploitation monopolistique protégée par le pouvoir), on passe progressivement à l'autorité reconnue aussi comme source de bienfait public, bienfait linguistique et plus largement culturel, processus qui va s'accentuant de la fin du règne de François ${ }^{\text {er }}$ en 1545-1546 (privilèges de Salel, de Rabelais) jusqu'à la fin du règne d'Henri III (avec les privilèges de Louis Le Roy en 1575, La Gessée en 1578, Lefèvre de La Boderie en 1581); c'est ce que l'on aimerait désigner comme la période du privilège humaniste [...] Le privilège devient alors une défense de la littérature. Et l'on comprend mieux pourquoi cet objet, qui ne protège en réalité que marginalement les intérêts juridiques et financiers de l'auteur (ou du libraire à qui le transport en a été fait), a pu avoir une telle importance dans la république des lettres et a pu figurer si ostensiblement sur les pages de titre. ${ }^{4}$

Pour renforcer le pouvoir légitimant de ce "lieu stratégique où s'institutionnalise la littérature ${ }^{5}$, nombre d'auteurs soulignent, dans les pages de leurs ouvrages, combien ils œuvrent pour le bien public. Une requête sur les expressions «bien public » ou « utilité publique», dans un corpus d'environ deux mille pièces de contact échangées par des auteurs (principalement poètes) entre 1549 et le milieu des années $1580^{6}$, nous donne accès à plusieurs occurrences :

4. Michèle Clément et Edwige Keller-Rahbé, "Introduction. Des droits pour l'auteur », in Privilèges d'auteurs et d'autrices en France (XVI $-X V I I^{e}$ siècles). Anthologie critique (Paris : Classiques Garnier, 2017), 43-45.

5. Clément et Keller-Rahbé, «Introduction. Des droits pour l'auteur », 46.

6. Ce corpus est accessible en ligne. Il s'agit de notre base de données « RéseauxPoètesXVI » (https:// sites.univ-lyon2.fr/ReseauxpoetesXVI/front/index.php) qui recense 2200 pièces dans lesquelles une soixantaine d'auteurs se citent les uns les autres entre 1549 (date de parution de La Deffence, et illustration 
- Jacques Peletier du Mans, dans l'« Apologię a Louis Meigręt Lionnoęs » (1550) affirme qu'il a de l'estime pour les lettrés qui par leurs inventions ou leurs traductions ont " anvie de profiter au bien public $»^{7}$ et il précise que c'est " an faveur du bien public » qu'il a décidé de " coucher [ses désaccords avec Meigret] en form $\notin$ de Dialogu£ $»^{8}$;

- en 1556, Louis Le Caron fait dire à Ronsard dans « Ronsard, ou de la poésie " que "de touts [sic] les genres des hommes qui ont jadis fait reluire à l'utilité publique quelque profession, quelque art ou quelque science digne d'immortelle mémoire, nous lisons les poètes avoir esté les premiers ${ }^{9}$;

- en 1563, dans une "Remonstrance a la Roine », André de Rivaudeau affirme que Théodore de Bèze ouvre pour le «bien publique ${ }^{10}$ de la France;

- Une dizaine d'années plus tard, en 1574, Guillaume Du Bartas se félicite de vivre des rentes de ses domaines et fait l'hypothèse que ses œuvres sont plus profitables que celles des poètes qui mettent en scène leur ardeur laborieuse comme prix de l'immortalité :

Mais tel, mon cher Remond, \& nuit \& jour se peine

Pour s'immortaliser dont, peut estre, la peine

Ne sert tant au public que mon Oisiveté. ${ }^{11}$

de la langue françoyse) et 1586 (année qui suit la mort de Ronsard et voit paraitre des Tombeaux en son honneur). Les liens entre auteurs sont interrogeables de multiples façons; en outre, des recherches plein texte sont possibles au sein de ces pièces dans lesquelles les auteurs se mettent en scène en lien les uns avec les autres.

7. Jaques Peletier du Mans, Dialogue de l'Ortografe e Prononciacion Françoese (1555) suivi de La Réponse de Louis Meigret, éd. Lambert C. Porter (Genève : Droz, 1966), 5. C’est l'auteur qui souligne.

8. Peletier du Mans, Dialogue de l'Ortografe, 8. C'est l'auteur qui souligne.

9. Louis Le Caron, Dialogues, éd. Joan A. Buhlmann et Donald Gilman (Genève : Droz, 1986), 260-261. C'est l'auteur qui souligne.

10. Jacques Pineaux, La Polémique protestante contre Ronsard (Paris : Didier, 1973), v. 837, 143. C’est l'auteur qui souligne.

11. Guillaume Du Bartas, "Sonet a F. Remond Conseiller au Parlement de Bourdeaus ", in La Muse Chrestiene de G. de Saluste seigneur Du Bartas, A Madame Marguerite de France Roine de Navarre (Bordeaux : Simon Millanges, 1574, Bibliothèque municipale de Lyon, Rés. 382667), v. 12-14, fol. V 3 vº. 
Ces affirmations consonnent avec la politique culturelle royale telle qu'elle s'exprime dans les privilèges, tant l'autopromotion des poètes engagée par Marot et intensifiée dans les décennies suivantes - notamment par les poètes de la «Brigade » - semble avoir rencontré d'échos favorables chez les têtes couronnées. Michèle Clément a souligné, en effet, que les poètes étaient les plus privilégiés des auteurs :

de cette situation d'exception des poètes dans le cadre des privilèges généraux et de favoritisme dans le cadre des privilèges simples, il ressort le fait bien établi que les poètes sont considérés comme les relais principaux du pouvoir royal. ${ }^{12}$

Ce sont sans doute des convergences collectives de discours en faveur de la reconnaissance du rôle social du poète qui ont permis de donner de la visibilité et de la légitimité aux poètes dont la contribution au "bien public» est désormais reconnue par les privilèges. Ainsi en va-t-il des plaidoyers de la génération Marot ou de la "Brigade». Toutefois, les occurrences que nous avons relevées ci-dessus montrent qu'autour de la notion d'« utilité publique » se trament aussi des divisions et s'établissent des hiérarchies.

En effet, notre enquête dans la base « RéseauxPoètesXVI » fait apparaître qu'au sein des textes qui mettent en scène les relations entre auteurs, la notion d'utilité publique est souvent mobilisée en contexte de tension. Dans les exemples cités, Peletier s'en remet à la notion de bien public dans un texte où il entre en tension avec Louis Meigret; chez Louis Le Caron, la notion de profit public permet de distinguer les poètes des autres catégories de lettrés ; André de Rivaudeau compare Théodore de Bèze et Ronsard à l'aune de leur participation au bien public, afin de valoriser Bèze et dénigrer Ronsard ;

C'est l'auteur qui souligne. Si l'idée d'un dur labeur nécessaire pour s'immortaliser peut renvoyer aux conseils de La Deffence et aux premières prises de position de Ronsard, celle d'un délassement utile peut être empruntée au Du Bellay des Regrets (sonnet 2). Pour une réflexion sur la vertu de la littérature envisagée au prisme de l'utile dulce horatien, voir Nathalie Dauvois, « Passe-temps et vertu de la littérature. Un modèle horatien ?", in Valeur des lettres à la Renaissance. Débats et réflexions sur la vertu de la littérature, éd. Pascale Chiron et Lidia Radi (Paris : Classiques Garnier, 2016), 193-207.

12. Michèle Clément, «Les poètes et leurs libraires au prisme du privilège d'auteur au XVIe siècle : la proto-propriété littéraire ", in Les Poètes français de la Renaissance et leurs "libraires ", éd. Denis Bjaï et François Rouget (Genève : Droz, 2015), 28. 
enfin, Du Bartas suggère que ses œuvres sont peut-être plus utiles au public que celles des poètes que Barthélemy Aneau a appelés les «immortaliseurs d'eux-mêmes $»^{13}$. En somme, ces auteurs introduisent l'idée qu'il y a des degrés différents de participation des lettres à l'utilité publique, de même que Lidia Radi et Pascale Chiron ont récemment souligné, dans l'introduction à l'ouvrage collectif Valeur des lettres à la Renaissance. Débats et réflexions sur la vertu de la littérature, que la vertu des lettres devenait, à la Renaissance, relative à la singularité des œuvres :

$[\ldots]$ ce «bien » que procure la littérature est bien plus équivoque qu'il n’y paraît : le bien n'est pas seulement synonyme de vertu, il est fait aussi du plaisir d'écrire ou de lire. [...] Que devient dans ce contexte ce que nous appelons la « vertu » de la littérature ? Le mot vertu ne s'interprète plus dans le sens de " qualité morale » reconnue de manière collective comme fin de la littérature. La vertu littéraire renvoie alors à ce que le texte produit de manière singulière chez son lecteur. On pourrait définir la vertu comme puissance d'action, « force » conformément à l'origine latine « virtus», qui serait à la fois une force potentielle, et une force exercée : une puissance et son actualisation. Ce sens permet de reconnaitre à la littérature la qualité d'une expérience singulière, aussi bien d'écriture que de lecture. [...] La «vertu» de la littérature dépendrait ainsi des intentions de l'auteur et de l'effet produit sur son lecteur, elle serait vertu « singulière » et non plus universelle comme le présuppose son sens moral. ${ }^{14}$

À l'instar de la vertu de la littérature, l'utilité publique des lettres pourrait donc être singulière, relative à une œuvre ou à un point de vue. De la sorte, la notion d'intérêt public nourrit les débats sur la valeur des œuvres, sert d'argument dans la rivalité entre lettrés et recouvre des enjeux de pouvoir.

13. Barthélemy Aneau, "Quintil horatian », in Joachim Du Bellay, La Deffence, et illustration de la langue françoyse (1549), éd. Jean-Charles Monferran (Genève : Droz, 2001), 360.

14. Pascale Chiron et Lidia Radi, "Introduction ", in Valeur des lettres à la Renaissance. Débats et réflexions sur la vertu de la littérature (Paris : Classiques Garnier, 2016), 11-13. 


\section{2) Jugement du lecteur et valeur de l'œuvre}

Dans le cadre de cette rivalité autour de la plus ou moins grande participation des auteurs au bien public, le rôle de juge peut être remis au lecteur. En effet, « la mention des apports au bien public [...] donne [...] corps à un horizon d'attente, à un ensemble social en train d'émerger, le lectorat, et participe donc à la construction des "publics" »", analysent Michèle Clément et Edwige KellerRahbé. C'est ainsi que la notion d'utilité ou de bien public apparaît souvent dans les dédicaces ou les "Aux lecteurs », textes dans lesquels l'opération de lecture est quasiment toujours liée à l'opération de jugement. Dans le « Au lecteur » de L'Olive, en 1550, Du Bellay s'adresse aux « lecteurs de bon jugement » et valorise les auteurs «qui legerement n'exposent leurs conceptions au publique jugement des hommes ${ }^{16}$. Lannée précédente, il avait habilement suggéré avoir emporté l'adhésion du lecteur de sa Deffence, comme l'a montré Philippe Desan :

Dans une phrase isolée (sans titre) qui sépare la dédicace au Cardinal Du Bellay du texte, Joachim demande aux lecteurs (pluriel) de différer leur jugement après la lecture : « L'autheur prye les Lecteurs différer leur jugement jusques à la fin du livre, \& ne le condamner sans avoir premierement bien veu \& examiné ses raisons ». Du Bellay anticipe un jugement avant même la lecture du texte, il introduit ainsi l'idée d'un épitexte apparemment actif circulant autour de son manifeste. À la fin de l'ouvrage, le lecteur trouve finalement un avis " Au lecteur" (singulier) - lecteur que Du Bellay considère désormais comme un ami ("Amy Lecteur... »). L'auteur se targue ainsi d'avoir séduit les lecteurs récalcitrants qui étaient tout prêts à le condamner. ${ }^{17}$

Mettre en scène l'accueil bienveillant du lecteur permet ainsi à Du Bellay de conférer de la valeur à son opuscule. Mais le lecteur Barthélemy Aneau refuse que son jugement soit forcé. En 1551, dans le Quintil horatian, il souligne que

15. Clément et Keller-Rahbé, « Introduction. Des droits pour l'auteur », 46.

16. Joachim du Bellay, La Deffence, et illustration de la langue françoyse \& L'Olive, éd. Jean-Charles Monferran et Ernesta Caldarini (Genève : Droz, 2007), 233-234. C'est l'auteur qui souligne. Sauf indication contraire, les œuvres de Du Bellay sont citées dans cette édition.

17. Philippe Desan, L'Imaginaire économique de la Renaissance (Paris : Presses de l'Université ParisSorbonne, 2002), 319. 
l'Angevin par "publicque impression » lui a communiqué la Deffence en le priant de « differer [s] on jugement $»^{18}$ et qu'après avoir bien lu, il peut maintenant bien juger, c'est-à-dire pointer toutes les faiblesses de l'ouvrage.

La lecture, qualifiée de positive ou négative, est donc comme un indicateur de la valeur conférée à l'œuvre. C'est la raison pour laquelle, dans les «Au lecteur », l'œuvre publiée est souvent présentée comme ayant déjà reçu un jugement positif de quelques lecteurs de choix. Ronsard affirme par exemple que ses vers ont reçu l'approbation du poète Jacques Bouju et de la princesse Marguerite : dans une ode de 1550, il dépeint en effet le premier «Vantant [s]on petit merite / Devant les toutvoians yeus / De la sage Marguerite $»^{19}$. Ailleurs, les auteurs présentent leurs œuvres comme un échantillon ou comme un test pour aller sonder le jugement de lecteurs qui, cette fois-ci, ne sont plus identifiés précisément : si le public apprécie, s'il en veut plus, l'auteur promet d'en fournir davantage. Ronsard affirme ainsi dans le « Au lecteur» des Quatre premier livres des Odes (1550) : je n'« affect[e] pour ce livre aucun titre de reputation, lequel [livre] ne t'est laché que pour aller découvrir ton jugement, affin de t'envoier après un meilleur combatant $»^{20}$. De même, en 1569, Scévole de Sainte-Marthe estime les vers de ses Premieres CEuvres « imparfaitz \& peu dignes d'estre presentez en public » mais il s'en remet au lectorat pour établir un diagnostic : «je [m’en] remets, Lecteur, à ton bon jugement », affirme-t-il, car "j'ai bien voulu en monstrer ces eschantillons à fin de m'esclercir, \& decouvrir si l'ouvrage contentera noz hommes $»^{21}$. Les dédicaces et les "Aux lecteurs» apparaissent ainsi comme un lieu privilégié de la négociation de la valeur de l'œuvre.

C'est par exemple la raison pour laquelle, nous semble-t-il, Ronsard n'insère d'adresse "Au lecteur» (quasiment) que dans les moments où la légitimité et la valeur de l'œuvre peuvent susciter des débats houleux : au moment de la parution des Odes, en 1550, quand — avec Du Bellay et quelques autres - il veut s'arroger une place de choix dans le champ poétique ${ }^{22}$; dans

18. Aneau, «Quintil horatian », 304.

19. Ronsard, Euvres complètes I, éd. Paul Laumonier (Paris : Droz, 1931), I, 10, v. 9-11, 122.

20. Ronsard, Euvres complètes I, 49.

21. Scévole de Sainte-Marthe, Euvres complètes I, éd. Jean Brunel (Genève : Droz, 2010), 213.

22. Quatre premiers livres des Odes (1550) : «Au lecteur », "Avertissement au lecteur », "Suravertissement» et « Breve exposition par I. M. P.». 
les années 1560, c'est-à-dire sous le feu des attaques des réformés ${ }^{23}$; et en 1572 , à l'occasion de la parution de sa Franciade ${ }^{24}$, promise depuis longtemps mais fournie inachevée et donc l'exposant à des critiques ${ }^{25}$. C'est sans doute qu'il lui faut, dans ces moments-là, considérer impérativement cette instance qu'est le lecteur et qui sanctionne la valeur de l'œuvre.

De la sorte, les périodes de conflits au sein du champ poétique semblent propices à observer les discours adressés par les poètes « aux lecteurs » et, audelà du cadre restreint des "Aux lecteurs », à analyser l'usage que ces poètes font du public et de la notion de « bien public » pour légitimer leurs œuvres ou se disputer les uns avec les autres. C'est dans ces moments d'affrontement que la construction des publics est certainement la plus saillante.

\section{Quelques usages du public dans les tensions poétiques des années 1550}

\section{L'argument des doctes et des pairs}

Du Bellay et Ronsard adoptent à leurs débuts un ton polémique pour faire sensation dans le monde des lettres, ton polémique qui repose sur des clivages, notamment en termes de public visé. Du Bellay affirme dans La Deffence (1549) ne pas vouloir s'adresser aux prétentieux dont la " sotte arrogance » rejette tout ce qui est écrit en français, mais s'adresser en revanche aux " sçavans ${ }^{26}$ - même un peu réticents - et au « lecteur studieux de la langue françoyse ${ }^{27}$ qui est le "Poëte futur ${ }^{28}$. Dans le « Au lecteur » de L'Olive de

23. Responce aux injures et calomnies (1563) : "Epistre ». Les trois livres du Recueil des nouvelles poesies (1564) : «Epistre au lecteur».

24. Les quatre premiers livres de la Franciade (1572) : «Au lecteur » et « Preface sur la Franciade, touchant le Poème heroïque. Au lecteur apprentif. ».

25. Ronsard insère à deux autres reprises un « Au lecteur » : une fois à l'ouverture de l'opuscule intitulé Discours a treshault et trespuissant Prince, Monseigneur le Duc de Savoye. Chant pastoral a Madame Marguerite, Duchesse de Savoye (Paris : Robert Estienne, 1559), et une autre fois à l'ouverture du Second livre des Meslanges (Paris : pour Vincent Sertenas, 1559). Dans les deux cas, il s'agit de préciser que les vers ont été écrits avant la mort du Roi Henri II dont le décès a occasionné un retard d'impression et que le lecteur doit donc les replacer dans leur contexte.

26. Du Bellay, La Deffence, et illustration, I, 1, 76.

27. Du Bellay, La Deffence, et illustration, II, 2, 127.

28. Du Bellay, La Deffence, et illustration, II, 3, 131. 
1549, il affirme ne pas vouloir prendre en compte ceux qui considèrent son nouveau style poétique « obscur, pource qu'il excede leur jugement $»^{29}$. Dans le « Au lecteur» de L'Olive augmentée (1550), il souligne l'opposition entre les «lecteurs de bon jugement $»^{30}$ et les « rimeurs ${ }^{31}$ qu'il méprise. Dans le même temps, Ronsard rejette dans le "Au lecteur » des Odes de 1550 le public des " rimeurs, \& principalement des courtizans $»^{32}$, qui sont des « ignorant $[\mathrm{s}] »$ et «petis lecteurs Poëtastres » de "peu de jugement» représentant une «vermine de gens ignorantement envieuse » à laquelle il oppose les « gentils espris, ardans de la vertu » auxquels il souhaite s'adresser.

Cette construction contrastée des publics se retrouve dans les discours que les deux ambitieux tiennent sur le lectorat des auteurs dont ils veulent minimiser la valeur poétique. C'est ainsi que Du Bellay écrit dans La Deffence à propos de Marot :

Ceux qui admirent voluntiers les petites choses, et deprisent ce, qui excede leur Jugement, en feront tel cas, qu'ilz voudront ; mais je sçay bien que les sçavans ne les mettront en autre Ranc, que de ceux, qui parlent bien Françoys, et qui ont [...] bon Esprit, mais bien peu d'Artifice. ${ }^{33}$

Il joue donc de l'opposition entre un public peu exigeant qui se satisfait des œuvres de Marot et un public exigeant qui reconnaît qu'on peut faire mieux. C'est aussi par le biais du jugement du public savant qu'il essaie de disqualifier Sébillet sans le nommer : c'est « chose grandement à reprendre, voyre odieuse à tout Lecteur de liberale Nature, voir en une mesme Langue une telle Immitation, comme celles d'aucuns sçavans mesmes, qui s'estiment estre les meilleurs, quand plus ilz ressemblent un Heroet, ou un Marot $»^{34}$. Le détour par le public lui sert enfin à discréditer Jean Le Blond, François Habert, François Sagon et Charles Fontaine lorsqu' il écrit : "O combien je desire voir secher ces Printems, chatier ces Petites Jeunesses, rabbattre ces Coups d'essay, tarir ces Fontaines, bref,

29. Du Bellay, La Deffence, et illustration, 355.

30. Du Bellay, La Deffence, et illustration, 234.

31. Du Bellay, La Deffence, et illustration, 234.

32. Ronsard, Euvres complètes I, 47-48.

33. Du Bellay, La Deffence, et illustration, II, 3, 128.

34. Du Bellay, La Deffence, et illustration, I, 8, 94. C'est l'auteur qui souligne. 
abolir tous ces beaulx tiltres assez suffisans pour degouter tout Lecteur sçavant d'en lire d'avantaige ! $»^{35}$. Par conséquent, le lectorat souhaité par Du Bellay est défini en creux : ce n'est pas le lectorat qui admire Marot, Sébillet, Habert et Fontaine ; le lectorat auquel il prétend est un lectorat qu'il qualifie de plus « savant».

Ce public n'est pas nombreux, semble-t-il, car Du Bellay double l'opposition docte / indocte de l'opposition vaste / restreint. À ses yeux, ce sont les poètes de peu de talent qui séduisent largement les publics. Il dénonce ainsi, dans La Deffence, les "Traditeurs " (mauvais traducteurs) qui « seduysent les lecteurs ignorans ${ }^{36}$. Il fustige, dans l'Olive augmentée, les «barbares poësies, qui par je ne scay quel nostre malheur plaisent communement plus aux oreilles françoises que les escritz d'antique \& solide erudition ${ }^{37}$. Il s'offusque également, dans le même ouvrage, des " escriz d'ung petit magister, d'un conard, d'un badault, \& aultres mignons de telle farine, dont les oreilles de nostre peuple sont si abbreuvées, qu'elles ne veulent aujourd'huy recevoir aultre chose $»^{38}$.

Une telle réflexion sur l'amplitude du public touché, qui vante les mérites d'une réception restreinte plutôt que large, et qui dédaigne le public ignorant, prend ses racines chez Horace (Satires, I, 4 et I, 10). À la manière du maître, les deux amis définissent leur ligne poétique au miroir de l'auditoire ou du lectorat recherché. En refusant de produire ce qui réussit auprès du public et qui est pratiqué par le plus grand nombre, en prônant une écriture exigeante pour un public rare de savants et d'amoureux de la langue française, Du Bellay et Ronsard ne cherchent pas à faire table rase, mais plutôt à esquisser les contours d'une sorte de "sous-champ de production restreinte " qu'ils occuperaient. Pour Pierre Bourdieu le «sous-champ de production restreinte où les producteurs n'ont pour clients que les autres producteurs" s'oppose au "sous-champ de grande production qui se trouve symboliquement exclu et discrédité ${ }^{39}$. Or, nous l'avons noté, Du Bellay et Ronsard prétendent viser un lectorat restreint de qualité. Par ailleurs, ils esquissent volontiers les contours d'un entre-soi élitiste en valorisant la lecture de quelques-uns de leurs pairs les plus éminents. C'est ainsi que, dans le «Au lecteur » de L'Olive (1549), Du Bellay prétend :

35. Du Bellay, La Deffence, et illustration, II, 11, 168.

36. Du Bellay, La Deffence, et illustration, I, 6, 89.

37. Du Bellay, La Deffence, et illustration, 233.

38. Du Bellay, La Deffence, et illustration, 233-234.

39. Pierre Bourdieu, « Le champ littéraire », Actes de la Recherche en Sciences sociales 89 (1991) : 7. 
« Il me suffit pour tous lecteurs avoir un S. Gelays, un Heroët, un de Ronsart, un Carles, un Sceve, un Bouju, un Salel, un Martin, et si quelques autres sont encor' à mettre en ce ranc. A ceulx là s'addressent mes petiz ouvraiges $»^{40}$. Si l'affirmation relève de la provocation, elle n'en construit pas moins un premier public, un public de choix composé de quelques personnes nommées, qui n'interdit sans doute pas - quoiqu'il en dise - un deuxième public, plus large et anonyme.

Évidemment, ceux qui sont relégués dans le "sous-champ de grande production " récusent cette répartition des rôles. Barthélemy Aneau, dans le Quintil horatian, qualifie d'hermétique la poésie trop érudite des nouveaux poètes. Et quand Du Bellay conseille «Sur toute chose, prens garde que ce Poeme soit eslongné du vulgaire ", Aneau répond que cela conduit à se couper de la communauté des hommes - en alléguant à son tour Horace :

Ceste caution est contre le precept d'Horace [Art poétique, v. 240-243] : qui veult le Poeme estre tel : que l'honneur d'iceluy soit acquis des choses, et parolles prinses au mylieu de la communauté des hommes, tellement que tout lecteur, et auditeur en pense bien povoir autant faire, et toutesfois n’y puisse advenir. Tel (à la verité) que ha esté Marot. Et toy au contraire, commandes de estranger la Poesie : disant que ne escris sinon aux doctes. ${ }^{41}$

Aneau suggère que Du Bellay, en « estrangeant » la Poésie et en prétendant ne s'adresser qu'aux doctes, ne participe pas vraiment au bien public. Parallèlement, le Lyonnais revalorise les œuvres de Fontaine et Habert en utilisant à son tour l'argument du public, mais pour souligner un effet sur ce public qui n'est pas du tout celui supputé par Du Bellay : « leurs tiltres [...] sont modestes, et non ambitieux comme le tien, ne degoustans les Lecteurs (comme tu dis), mais plustost les invitant $»^{42}$.

Cette ouverture généreuse au public - et potentiellement à un large public - est également ce que valorise Fontaine lorsqu'il prend la défense de Marot dans une adresse "Au lecteur» ouvrant le recueil Sensuyvent les Ruisseaux de Fontaine (1555):

40. Du Bellay, La Deffence, et illustration, 355-356.

41. Aneau, «Quintil horatian », 342.

42. Aneau, «Quintil horatian », 358. 
«Au lecteur»

[...]

Marot pour sa facilité

Sera leu, comme il a esté,

Du commun (auquel ne veulx plaire

Totalement, n'aussi desplaire)

Toujours aura plus de lecteurs

Que cent, \& cent, d'autres auteurs. ${ }^{43}$

Il s'agit donc bien d'un conflit à propos de la valeur des lettres dans lequel intervient l'argument du lectorat. Dans un camp, un lectorat restreint d'élite est censé refléter la haute valeur de l'œuvre ; dans l'autre camp, un vaste lectorat est censé démontrer la participation généreuse de l'œuvre et de son auteur au bien commun.

Dans le même temps, une autre stratégie se développe chez les adversaires de la nouvelle génération, stratégie qui consiste à renverser l'attribution de ces places au sein du champ poétique, en faisant glisser les nouveaux poètes $\mathrm{du}$ "sous-champ de production restreinte " vers le « sous-champ de grande production ». En effet, comme l'ode et le sonnet amoureux deviennent rapidement à la mode, l'audace et la nouveauté que réclamaient pour eux les jeunes ambitieux perdent de leur pertinence. Du Bellay et Ronsard se plaignent d'ailleurs assez vite d'être trop massivement et trop maladroitement imités ${ }^{44}$.

Pour ce qui concerne l'ode, Jacques Tahureau, allié des nouveaux poètes, s'attache par exemple à en défendre la nouveauté et la singularité dans la dédicace de ses Premières Poësies de 1554 au cardinal de Guise. Selon lui,

43. Charles Fontaine, "Au lecteur", in Sensuyvent les Ruisseaux de Fontaine : Euvre contenant Epitres, Elegies, Chants divers, Epigrammes, Odes, \& Estrennes pour ceste presente annee 1555. Par Charles Fontaine, Parisien. Plus y a un traité du passetems des amis, avec un translat d'un livre d'Ovide, \& de 28 Enigmes de Symposius, traduits, par le dict Fontaine (Lyon : Thibauld Payan, 1555, Bibliothèque nationale de France Rés. Ye-1610), v. 13-18, 207-208.

44. Par exemple, Ronsard dans la célèbre « Élégie de P. de Ronsard, à Chretophle de Choiseul, Abbé de Mureaux » de 1556, Euvres complètes VIII, éd. Paul Laumonier (Paris : Nizet, 1935), v. 15-24, 352-353 : «Du regne de HENRY, cinq ou six seulement, / Vindrent, qui d'un acord moderé doucement, / Et d'un pouce atrempé firent doctement bruire / Maintenant la guitterre, et maintenant la lyre, / Et maintenant le luc, et oserent tenter / Quelque peu la trompette affin de haut chanter. / Incontinent apres, une tourbe inconnue / De serfz imitateurs, pesle mesle est venue / Se ruer sans esgard, laquelle a tout gasté / Cela que les premiers avoient si bien chanté ». 
pour minimiser la pertinence de l'appel lancé par Du Bellay dans La Deffence ("Chante moy ces Odes, incongnues encor' de la Muse Françoyse », II, 4) et l'affirmation de nouveauté de Ronsard («Quand tu m'appelleras le premier auteur Lirique François, \& celui qui a guidé les autres au chemin de si honneste labeur, lors tu me rendras ce que tu me dois ${ }^{45}$ ), les ignorants veulent
trancher le chemin à tous les bons espriz de travailler en nostre poësie Françoyse, et principalement en l'Ode, l'une de ses plus industrieuses parties, alleguans cela avoir desja esté assez traicté par d'autres, comme s'ilz nous vouloyent faire trop vulgaire et commun ce qui ne faict encores que commencer à naistre en nostre France. ${ }^{46}$

Il songe sans doute à Barthélemy Aneau, entre autres, qui a récusé que l'ode soit si « incongnue » que ne le prétendaient Du Bellay et Ronsard ${ }^{47}$. En cette période de débat sur la nouveauté ou l'ancienneté de l’ode - dont Jean-Charles Monferran a dénoué quelques fils pour montrer la "supercherie » de Ronsard visant à « dessin[er] une ligne de partage entre un avant et un après, entre "une antiquité" et une modernité $»^{48}-$ Tahureau cherche alors à maintenir l'ode dans un champ de production nouvelle et restreinte : elle n'est pas encore populaire, elle reste une nouveauté et la pratiquer demeure un moyen de se distinguer. Quoique l'argument du public ne soit pas explicitement convoqué, l'idée de production restreinte sous-tend l'affirmation de Tahureau et suppose sans doute également un lectorat trié sur le volet, à même d'apprécier pleinement la nouveauté d'un genre antique (illustré par Horace et Pindare) restauré à la française.

Quant à la poésie amoureuse, elle est en vogue dans les années 1550, comme l'ont par exemple montré Cécile Alduy ou Daniele Maira en analysant

45. Ronsard, Euvres complètes I, 43.

46. Jacques Tahureau, Poésies complètes, éd. Trevor Peach (Genève : Droz, 1984), 61.

47. Aneau, "Quintil horatian », 341 : «Vray est que le nom Ode ha esté incogneu, comme peregrin, et Græc escorché et nouvellement inventé entre ceux qui en changeant les noms cuydent deguyser les choses : mais le nom de chant et chanson est bien cogneu, et receu comme François. »

48. Jean-Charles Monferran, « À propos de la constitution du genre de l'ode : les définitions de l'ode française avant Ronsard ", dans Renaissance de l'Ode. L'Ode française au tournant des années 1550, éd. Nathalie Dauvois (Paris : Honoré Champion, 2007), 46. 
les phénomènes de «collection » des recueils d'Amours ${ }^{49}$ auxquels participent pleinement les poètes de la nouvelle génération (Du Bellay, Ronsard, Des Autels, Magny, etc.). Ceux qui pratiquent ce genre à la mode, décliné parfois dans des versions lascives, sont alors accusés de verser dans la facilité. Jean Macer ${ }^{50}$, dans une Philippique contre les Poëtastres et rimailleurs françois de nostre temps (Paris : Guillaume Gaillard, 1557) - que Marcel Raymond avait découverte et publiée en extraits en $1926^{51}$ - reproche ainsi aux poètes de séduire ${ }^{52}$ les publics en flattant leurs bas instincts : " on se commence à saouler de vos folatries", écrit-il. Sans doute vise-t-il en premier lieu les Folastries de Ronsard mais aussi plus largement la vogue des canzonieri. Le « on » suppose l'agacement d'un public exigeant. Le discours de Macer peut alors être lu comme un décalque du discours de Du Bellay sur les « mignons de telle farine, dont les oreilles de nostre peuple sont si abbreuvées ${ }^{53}$. Comme Du Bellay au début des années 1550, Jean Macer discrédite les poètes qui séduisent les publics en versant dans la facilité et suggère une saturation face à ce type de production. De la sorte, il sape les prétentions des nouveaux poètes à être singuliers, peu nombreux et élitistes, même si dans les faits Ronsard, Du Bellay, Baif et leurs amis essaient sans cesse de se renouveler pour ne pas s'enraciner dans des genres qui, devenant à la mode, perdent de leur audace.

Si le concept bourdieusien de "sous-champs » de grande production et de production restreinte nous a semblé utile dans le cadre des affrontements entre auteurs, nous n'en sommes pas moins consciente que cette notion, forgée pour le monde littéraire du XIX siècle, ne peut être transférée au XVI ${ }^{\mathrm{e}}$ siècle que moyennant prudence et aménagements. Si la poésie peut avoir des

49. Cécile Alduy, Politique des « Amours ». Poétique et genèse d'un genre français nouveau (1544-1560) (Genève : Droz, 2007) ; Daniele Maira, Typosine, la dixième muse. Formes éditoriales des canzonieri français (1544-1560) (Genève : Droz, 2007).

50. Marcel Raymond l'a identifié comme étant possiblement l'imprimeur juré Jean Macé ; Jean-Eudes Girot le qualifie de "Catholique partisan d'une réaction morale » dans Pindare avant Ronsard. De l'émergence du grec à la publication des Quatre Premiers livres des Odes (Genève : Droz, 2002), 371.

51. Marcel Raymond, "Deux pamphlets inconnus contre Ronsard et la Pléiade », Revue du Seizième Siècle 13 (1926) : 243-264.

52. « [S]éduire n'est autre chose qu'avec baverie, tromperie et chose fabuleuse, decevoir autrui » affirme Jean Macer aux poètes qu'il qualifie de «lascif[s] » et « lubrique[s] ».

53. Du Bellay, "Au lecteur » de L'Olive augmentée (1550), in La Deffence, et illustration de la langue françoyse \& L'Olive, 233-234. 
velléités d'autonomie à la Renaissance ${ }^{54}$, le processus d'autonomisation du " champ littéraire » n'en est qu'à ses balbutiements. La production poétique n'est notamment pas émancipée du « champ » politique. Certes, ici les poètes, et notamment ceux de la génération 1550, peuvent se targuer de n'écrire que pour une poignée de confrères exigeants, mais là ils se flattent aussi abondamment d'être lus par les princes et les rois, tant ce lectorat est à même de conférer une haute valeur à leurs œuvres.

\section{2) L'argument des rois et des princes}

C’est en effet un procédé courant sous la plume de Ronsard, de Du Bellay et de leurs amis d'affirmer qu'ils sont lus et appréciés du roi, de la reine, de tel prince ou de telle princesse. Ronsard vante « [s]a chanson sucrée / Qui les grands Princes recrée » dans l'ode 11 du livre I de ses Quatre premiers livres des Odes $(1550)^{55}$. Du Bellay, dans la dédicace de son Recueil de poesie de 1549 à Marguerite, affirme que la sœur de Henri II l’a reçu et que, lors de cette entrevue, il a « cogneu [s]es petiz labeurs [lui] avoir esté agreable » ${ }^{56}$, ce qui, par conséquent, le dispense de répondre à ses détracteurs :

Je ne me veulx amuser ici à respondre aux calumniateurs (comme est la façon ordinaire des ecrivains) puis que mes escriz ont desja esté si heureux de rencontrer la faveur de vostre jugement, \& par vostre moyen celuy du Roy, \& de la Royne, auxquels ayant satisfaict, tant s'en fault que je me soucie du mescontentement d'autruy. ${ }^{57}$

54. Voir Caroline Trotot, « La détermination du champ littéraire entre rhétorique et poétique autour de la Pléiade ", in Constitution du champ littéraire. Limites, intersections, déplacements, éd. Pierre Chiron et Francis Claudon (Paris : L’Harmattan, 2008), 191-207 ; Jean-Charles Monferran, L'École des Muses. Les arts poétiques français à la Renaissance (1548-1610). Sébillet, Du Bellay, Peletier et les autres (Genève : Droz, 2011) ; Florence Bonifay, " "ReseauxPoetesXVI" : une base de données pour étudier les réseaux de poètes au XVI ${ }^{\mathrm{e}}$ siècle », in Le Réseau. Usages d'une notion polysémique en sciences humaines et sociales (Louvain-la-Neuve : Presses Universitaires de Louvain-la-Neuve, 2016), 27-42.

55. Ronsard, Euvres complètes I, v. 15-16, 127.

56. Du Bellay, Euvres poétiques III, éd. Henri Chamard (Paris : Nizet, 1983 [1912]), 58.

57. Du Bellay, Euvres poétiques III, 59. 
Se targuer d'un lectorat princier et royal dont l'appréciation est positive est évidemment un argument de poids quant à la valeur de l'œuvre. Tahureau recourt au même procédé lorsqu'il présente ses Premieres poësies (1554) comme déjà appréciées par la sœur du roi Henri II et par le cardinal de Guise :

[...] ayant desja tant receu d'heur et d'honneur en la Court de nostre Roy, que d'y avoyr, d'entre les meilleurs et plus doctes jugementz, peu contenter celuy de la plus sçavante et admirable de toutes les Princesses, sœur du premier de tous les Roys, la premiere Marguerite, laquelle m’ha donné tant de courage et d'espoir [...] qu'il me semble ne pouvoir presque faillir à faire quelque chose de bon estant advoué d'une si parfaite et divinement rare excellance, et de vous, Monseigneur. ${ }^{58}$

Ces cautions prestigieuses sont un enjeu de pouvoir, de sorte que l'on comprend pourquoi Ronsard ne souhaite à ses détracteurs qu'une chose dès 1549, qui est de n'arriver jamais à être lus et appréciés par les rois :

\footnotetext{
«L'Hymne de France»

[...]
}

Moy ton Poëte, ayant premier osé

Avoir ton loz en ryme composé,

Je te supply, qu'à gré te soit ma lyre :

Et si quelcun enraige d'en médire,

Soit-il prisé de l'obscur populaire,

Et ses labeurs ne puissent jamais plaire

A deux ou trois, l'honneur de ta province,

Ny aux saincts yeux de mon grand Roy, ton Prince. ${ }^{59}$

On retrouve une construction binaire des publics : le public non qualifiant qu'est l'« obscur populaire » (nombreux) — que Ronsard dédaigne à l'instar d'Horace et des poètes alexandrins ${ }^{60}$ - contre le public qualifiant qu'est le Roi (unique).

58. Tahureau, «A Monseigneur Le Reverendissime Cardinal de Guyse », in Poésies complètes, 62.

59. Ronsard, Euvres complètes I, v. 217-224, 35.

60. Voir les pages de Bénédicte Delignon sur le topos alexandrin de l'ignorance de la foule et du poète incompris du vulgaire dont s'inspire Horace : Bénédicte Delignon, Les Satires d'Horace et la comédie 
Il y a moins de controverses possibles à propos du lectorat princier et royal qu'à propos du public large ou restreint, ignorant ou docte, que les poètes s'arrogent ou se refusent mutuellement. Lorsqu'un poète se targue d'avoir été lu et apprécié par la princesse Marguerite, il semble difficile de lui refuser ce lectorat ou de le disqualifier (il serait audacieux d'affirmer que la princesse n’a pas de goût!). De la sorte, si les poètes sont évidemment en rivalité pour afficher leur proximité avec les grands, ce public-là est toutefois l'objet de peu de controverses. Une position nous semble toutefois surprenante : celle du «mélancolique » dans les Discours non plus mélancoliques que divers (Poitiers : Enguilbert de Marnef, 1556) ${ }^{61}$. Au chapitre premier, le mélancolique s'en prend aux « historiens qui cherchent l'origine des Gaulois \& François ». Il se moque notamment de ceux qui « ont tiré du sang de ces gentils de Troiens [sic], voire maugré nature, non seulement les Françoys, [...] mais aussi nos grans peres les Gaulois »; "Est-ce pas cela songé bien creus ? »" fantasmer une réception violente :

Que pleust au bon Dieu, lequel est par le bon Hebrieu appellé le Dieu des Dieus, que ces beaus jaseurs eussent presché telles origines en nostre Gaule, au temps que les bons Druides y avoient credit : vous eussiés veu en mon avis, qu'on eust fait de gentils sacrifices de leur cervelle (s'ils en ont les bons seigneurs). ${ }^{63}$

gréco-latine : une poétique de l'ambiguité (Paris, Louvain : Peeters, 2006), 387-393.

61. L'attribution de ce texte est encore en discussion. Voir Marie-Luce Demonet, " La singularité lyonnaise, vue d'ailleurs ", in Lyon et l'illustration de la langue française à la Renaissance, éd. Gérard Defaux (Lyon : ENS éditions, 2003), 345-360. Plusieurs hypothèses d'auteurs sont avancées : Jacques Peletier du Mans, Élie Vinet, Bonaventure Des Périers, Jean Boiceau de La Borderie, Jacques Tahureau. Voir trois autres articles qui analysent ce texte dans Les Grands Jours de Rabelais en Poitou, éd. MarieLuce Demonet (Genève : Droz, 2006) : Véronique Zaercher, "L'écriture à “diverses mains" dans les Discours non plus mélancoliques que divers ", 341-357; Sophie Arnaud, "Peut-on attribuer à Jacques Peletier du Mans la paternité des Discours non plus mélancoliques que divers des choses qui appartiennent à notre France? ", 359-378; Marie-Luce Demonet et Toshinori Uetani, " "Rabelaiseries" : la présence de Rabelais dans les Discours non plus mélancoliques que divers (1556) », 379-413.

62. Discours non plus melancoliques que divers, de choses mesmement, qui appartiennent a notre France: \& a la fin La manière de bien \& justement entoucher les Lucs \& Guiternes (Poitiers : Enguilbert de Marnef, 1557, Bibliothèque nationale de France Res-Z-1110), 3.

63. Discours non plus melancoliques que divers, 3 . 
D’ailleurs, conclut-il : «Ce seroit un mout grand bien pour la chose publique, que ces gentils escrivans eussent aussi belle envie de se taire \& repouser, que de mettre tels songes par escript : pour monstrer qu'ils sçavent je ne sçai quoi de bon plus que les autres ${ }^{64}$. Si le mélancolique vise le projet de la Franciade que Ronsard promet depuis $1550^{65}$, et dont il a dit en 1554 que le roi lui avait commandé de le mener à bien ${ }^{66}$, alors il suggère peut-être que le roi n'est pas bon juge des projets littéraires de Ronsard et, plus généralement, des écrits qui sont à même de contribuer au bien public. Quand bien même Ronsard est un auteur privilégié du roi depuis $1554^{67}$, le mélancolique refuserait de porter la lecture approbatrice d'Henri II au crédit de l'œuvre du Vendômois.

Le recours des poètes à des garantes de la qualité de l'œuvre que sont notamment les princesses nous conduit à observer, pour finir, quel usage il peut être fait du lectorat féminin dans le cadre de la valorisation ou de la dévalorisation des œuvres.

\section{3) L'argument des femmes}

Lorsque, dans La Deffence, Du Bellay conseille à l'apprenti poète de viser le genre poétique le plus haut, à savoir le poème épique, il oppose ce genre - viril - aux romans pour femmes : « Je veux bien en passant dire un mot à ceulx, qui ne s'employent qu'à orner et amplifier notz Romans, et en font des livres certainement en beau, et fluide langaige, mais beaucoup plus propres à bien entretenir Damoizelles, qu'à doctement écrire ${ }^{68}$. Au lectorat des demoiselles conviendraient donc des œuvres faciles à lire; mais comme le poète ambitieux doit viser une écriture savante et exigeante, ce lectorat ne serait pas sa cible.

64. Discours non plus melancoliques que divers, 4. C'est l'auteur qui souligne.

65. Voir l'« Ode de la Paix » adressée à Henri II en 1550 dans Ronsard, Euvres complètes III, éd. Paul Laumonier (Paris : Hachette, 1921), 3-35.

66. Voir l'« Elegie à Cassandre " dans Le Bocage (1554), in Ronsard, CEuvres complètes VI, éd. Paul Laumonier (Paris : Hachette, 1930), v. 5-8, 56-57 : «C'est lui qui veut qu'en trompette j'échange / Mon luc, afin d'entonner sa louange, / Non de lui seul, mais de tous ses aïeus / Qui sont issus de la race des Dieus ». 67. Daniele Maira, « Ronsard surintendant éditorial ou la genèse des Euvres (1554-1556) », Seizième Siècle 10 (2014) : 53 : «Ronsard obtient un privilège royal, signé à Fontainebleau le 4 janvier 1553 (1554 n. s.). Ce privilège est extraordinaire puisqu'il est délivré pour toutes les œuvres de Ronsard, celles qui ont déjà paru ainsi que celles qu'il doit encore écrire. C'est un privilège personnel, à durée illimitée ».

68. Du Bellay, La Deffence, et illustration, II, 5, 139. 
Toutefois, ni Du Bellay ni Ronsard ne peuvent négliger le lectorat féminin. La protection de la princesse Marguerite leur est nécessaire, leurs vers amoureux peuvent trouver une réception favorable chez un public féminin plus large et la vertu de leur poésie peut participer à leur édification. L'année même où paraît La Deffence, Du Bellay adresse ainsi son Recueil de poésie (qui n'est pas une épopée) à la sœur du roi Henri II, de sorte qu'il doit prendre en compte un lectorat féminin sans toutefois renoncer à la poésie docte qu'il a prônée dans La Deffence. Il délègue alors à son compatriote angevin Jean Proust le soin de rédiger des commentaires de ses vers pour en éclaircir le sens auprès des Dames. Dans son «Au lecteur », Jean Proust le stipule :

Je n’ay (lecteur) entrepris ce petit labeur pour enseigner Minerve [...]. Mais voulant satisfaire au plaisir, \& contentement de plusieurs bons jugemens, non toutefois exercitez en la lecture des poëtes, et singulierement pour soulaiger l'honneste labeur des dames, et damoizelles, qui voluntiers aiment à lire choses exquises, et non vulgaires. ${ }^{69}$

De la même manière, c'est quand il s'adresse à Marguerite, dans l'ode 3 du livre V (1552), que Ronsard prétend lui aussi à un lectorat féminin dont la vertu serait développée par la lecture de ses vers, notamment ceux qui chantent la Princesse :

Je publiray parmy la France

Le loz de ta divinité,

Tes vertus, bontez, \& doctrine,

[...]

Affin qu'apres ma voix fidele

Au soir à la tarde chandelle

Les meres faisans œuvres maintz,

Content tes vertuz precieuses,

A leurs filles non ocieuses,

Pour tromper le temps, \& leurs mains. ${ }^{70}$

69. Jean Proust, « Brieve exposition de quelques passaiges poëtiques les plus difficiles contenuz en cet œuvre ", in Recueil de poésie, présenté à tresillustre princesse ma Dame Marguerite, seur unique du Roy, et mis en lumière par le commandement de madicte dame (Paris : Cavellat, 1549), 68.

70. Ronsard, Euvres complètes III, v. 302-304 et v. 307-312, 114-115. 
Comme Paul Laumonier le souligne en commentaire de ces vers, " Ronsard, en dépit de ses rodomontades, désirait être lu du grand public, et même du "simple populaire", autant que des érudits et [...] il tenait à une gloire vraiment nationale $»^{71}$. En outre, ici, le lectorat féminin dont il se targue, composé des mères vertueuses, apparait comme un gage de son écriture vertueuse.

Toutefois, après la parution des Amours de Meline de Baif, dont le deuxième livre est assez leste, après la publication de vers ronsardiens célébrant la pompe du bouc de Jodelle et après la sortie du Livret de Folastries, la question du lectorat féminin devient un argument des adversaires de la Brigade pour dévaloriser les œuvres de ces derniers. Tahureau témoigne lui-même de la réception scandalisée ${ }^{72}$ qu'ont pu provoquer les Amours de Baiff dans le poème intitulé «A une damoyselle, qui brulla les Amours de J.A. de Baif, pour le sonnet "O doux plaisir", etc » (Les Premieres poësies, 1554). Il le fait sans animosité contre Baif, dont il prend au contraire la défense. En revanche, Jean Macer dans la Philippique contre les Poëtastres et rimailleurs françois de nostre temps, utilise le scandale pour discréditer les jeunes poètes amoureux et tenter de leur retirer le lectorat féminin. C'est, pour lui, faire « $[\mathrm{s}]$ on devoir envers le bien public $»^{73}$ que de dénoncer le paganisme et la lubricité. Il interdit alors aux femmes et aux jeunes gens de lire ces vers : «Fuyez, fuyez, jeunes pucelles, et vous adolescens, auxquelz raison est encore chambriere non emancipée, telz escripts execrables $»^{74}$. De manière contradictoire, mais sans doute pour discréditer plus sévèrement encore ses adversaires, il affirme que de toutes manières les femmes ne lisent pas ou ne lisent plus les poésies amoureuses lascives :

Vous congnoissez quasi qu'on se commence à saouler de vos folatries, veu
que mesme le temps qui court nous a amené ce bonheur, que les dames
Françoyses bien instruites sont totalement soliciteuses et soigneuses de
chansons salutaires, qui ensemble repaissent le corps et l'esprit : combien
qu'un peu devant l'eussent vos folies et parolles palies de Venus; mais en

71. Ronsard, Euvres complètes III, 115.

72. Jacques Tahureau, Poésies complètes, éd. Trevor Peach (Genève : Droz, 1984), 215-217.

73. Jean Macer, Philippique contre les Poëtastres et rimailleurs françois de nostre temps (1557), in Marcel Raymond, "Deux pamphlets inconnus contre Ronsard et la Pléiade ", Revue du Seizième Siècle 13 (1926) : 253 .

74. Macer, Philippique contre les Poëtastres, 254. 
la fin ont cogneu que telles baguenauderies detournent plustost l'esprit de vertu que l'induire. ${ }^{75}$

Refuser le public qualifiant des femmes vertueuses aux adversaires est une manière de disqualifier, encore une fois, les œuvres qu'ils produisent.

\section{Conclusion}

En somme, au cœur de ce XVI e siècle caractérisé par une " augmentation considérable de la production et, corrélativement de la consommation littéraire ", au cours duquel " la multiplication du nombre des lecteurs, et des auteurs qui cherchent à satisfaire leur curiosité $[\ldots]$ instaure de nouveaux rapports entre l'écrivain et un public qui se diversifie » ${ }^{76}$, les années 1550 offrent un terrain d'exploration particulièrement intéressant des représentations des publics par les auteurs, dans la mesure où l'arrivée d'une nouvelle génération poétique ambitieuse veut discuter de la valeur des œuvres passées et à venir au prisme de leur lectorat. Dans ce cadre-là, entre les novateurs et leurs opposants, la figure du public est instrumentalisée pour attribuer ou refuser une valeur à l'œuvre. Les qualificatifs accolés au lectorat (large, restreint, docte, inculte, royal, féminin, etc.) sont l'occasion de définir des publics souhaités - parce que qualifiants - et des publics refusés - parce que non-qualifiants. Si ces constructions ne nous renseignent pas avec précision sur la réalité des publics, la multiplicité des qualificatifs témoigne en revanche d'une appréhension différenciée et hiérarchisée des publics. L'échelle des publics ne fait toutefois pas consensus puisque certains lectorats, sous la plume des poètes qui se disputent, ont une valeur réversible (les femmes ou le « grand public » par exemple).

Mais par-delà la valeur symbolique de ces publics construits par les auteurs, s'entrevoit évidemment la valeur économique de marchés à conquérir. Certains poètes tentent donc de concilier aspirations élitistes et nécessité de ne pas s'aliéner le marché du public plus populaire. Ici Ronsard dit vouloir emprunter une voie étroite : je " Ne suis ni le sens, ni la rime, / Ni l'art, du moderne ignorant, / Bien que le vulgaire l'estime, / Et en béant l'aille adorant ${ }^{77}$.

75. Macer, Philippique contre les Poëtastres, 250.

76. Charles Adelin Fiorato et Jean-Claude Margolin, «Introduction », in L'Écrivain face à son public en France et en Italie à la Renaissance (Paris : Vrin, 1989), 7.

77. Ronsard, Euvres complètes I, II, 21, v. 13-16, 237. 
Là, malgré ses proclamations hautaines et son mépris affiché du vulgaire dans le «Au lecteur » des Odes (1550), il stipule dans l'« Avertissement au lecteur » qu'il s'adresse à un double public, ce qui explique son inconstance orthographique : « cherchant tous les moiens que je puis de servir aus oreilles du sçavant, \& aussi pour acoutumer le vulgaire à ne regimber contre l'éguillon, lors que on le piquera plus rudement $\aleph^{78}$. Quelques années plus tard, il fait état de son incompréhension face aux critiques de publics auxquels il a pourtant tenté de s'adapter $^{79}$. Les poètes semblent ainsi tâtonner, à l'heure où ils font leur entrée sur le marché du livre, entre construction d'un public idéal comme indicateur de la valeur de l'œuvre et prise en compte d'un public réel à conquérir.

78. Ronsard, Euvres complètes I, 53-54. C'est l'auteur qui souligne.

79. Ronsard, Euvres complètes VII, éd. Paul Laumonier (Paris : Didier, 1959), v. 1-8, 115-116 : «Thiard, chacun disoit à mon commencement / Que j'estoi trop obscur au simple populaire : / Aujourd'hui, chacun dit que je suis au contraire, / Et que je me dements parlant trop bassement. / Toi, qui as enduré presqu'un pareil torment, / Di moi, je te suppli, di moi que doi-je faire ? / Di moi, si tu le sçais, comme doi-je complaire / A ce monstre testu, divers en jugement?» 